\title{
Chronic Biliary Obstruction Induces Pulmonary Intravascular Phagocytosis and Endotoxin Sensitivity in Rats
}

\author{
Shih-Wen Chang and Narumi Ohara \\ Pulmonary and Critical Care Division, Department of Medicine, Northwestern University Medical School and Veterans Administration \\ Lakeside Medical Center, Chicago, Illinois 60611
}

\begin{abstract}
Endotoxin sensitivity varies among animal species and appears to correlate with the presence of pulmonary intravascular macrophage (PIM). In rats, which lack PIM, we investigated the hypothesis that chronic cholestatic liver injury leads to induction of PIM and endotoxin sensitivity. Rats were randomized to either common bile duct ligation (BDL) or sham-surgery and studied at 1 wk (acute cholestasis), 2 wk (cholestasis, early cirrhosis), and 4 wk (cholestasis, established cirrhosis) after surgery. Intravascularly injected fluorescent latex microspheres ( $1 \mu \mathrm{m}$ diameter) were taken up by large phagocytic cells in lung parenchyma of BDL rats (at 2 and 4 wk), while no uptake was observed in lungs from control rats. Electronmicroscopy revealed accumulation of large, mononuclear, macrophage-like cells containing ingested latex particles within the pulmonary capillaries. Pulmonary intravascular phagocytosis, as reflected in lung uptake of ${ }^{99 \mathrm{~m}}$ Tc microaggregated albumin (Microlite, mean particle diameter $=1 \mu \mathrm{m})$, averaged $0.7 \pm 0.1 \%$ ( $m e a n \pm S E M)$ of total injected dose in 13 control rats and progressively increased with time after BDL (1 wk, $1.7 \pm 0.2 \%$; 2 wk, $10.0 \pm 3.0 \%$; 4 wk $35.1 \pm 5.9 \%$ ). Rats with biliary cirrhosis were markedly sensitive to the lethal effects of low dose endotoxin and demonstrated marked lung edema at the time of death. Furthermore, the lung uptake of intravascular ${ }^{125} \mathrm{I}$-lipopolysaccharide was increased fivefold in cirrhotic rats. We conclude that chronic biliary obstruction leads to the induction of pulmonary intravascular phagocytes and enhances endotoxin sensitivity in rats. Pulmonary intravascular phagocytosis in patients with advanced cirrhosis may account for their increased susceptibility to sepsis-induced adult respiratory distress syndrome. (J. Clin. Invest. 1994. 94:2009-2019.) Key words: liver cirrhosis - bile duct ligation - pulmonary intravascular macrophage $\cdot$ sepsis $\cdot$ lung injury
\end{abstract}

Preliminary reports of this study were presented in abstract form at the Annual Meetings of the American Thoracic Society, May 1990 (Am. Rev. Respir. Dis. 141:A644) and May 1991 (Am. Rev. Respir. Dis. 143:A333).

Address correspondence to Shih-Wen Chang, MD, Mercy Health System, 1000 Mineral Point Avenue, P.O. Box 5003, Janesville, WI 535475003.

Received for publication 22 February 1993 and in revised form 15 June 1994.

J. Clin. Invest.

(C) The American Society for Clinical Investigation, Inc.

$0021-9738 / 94 / 11 / 2009 / 11 \$ 2.00$

Volume 94, November 1994, 2009-2019

\section{Introduction}

Sensitivity to the toxic effects of bacterial LPS, or endotoxin, varies by several orders of magnitude across animal species (1). Certain species, such as sheep, calf, or pig, are exquisitely sensitive, responding to microgram quantities of endotoxin with shock, pulmonary hypertension, increased-permeability lung edema, and death (1-3). Other species, such as rat, dog, and monkey, can tolerate milligram quantities of endotoxin with only moderate systemic hypotension $(1,4-6)$. Even when given a lethal dose of endotoxin, their pulmonary vascular responses are relatively mild and significant lung edema is often difficult to demonstrate (4-7). The reason for this marked species difference in endotoxin sensitivity is unclear and cannot be easily explained on the basis of differences in the synthesis or release of cytokines or lipid mediators or in the response of circulating inflammatory cells to endotoxin.

Recently, several authors have suggested that the presence of pulmonary intravascular macrophage (PIM) ${ }^{1}$ accounts for the marked species-dependent sensitivity to endotoxin-induced lung injury (8-10). PIMs are large mononuclear phagocytes which reside in the pulmonary microcirculation of certain animals and which resemble the hepatic Kupffer cells in both morphology and function. Lungs of endotoxin-sensitive species, such as sheep, calf, and pig, contain large numbers of PIMs and avidly take up intravascular bacteria, endotoxin and other particulates $(8,11)$. In contrast, lungs of endotoxin-resistant species, such as rats and dogs, are characterized by very few or no PIMs and minimal uptake of intravascular particulates or endotoxin. Phagocytosis of bacteria or endotoxin by PIM is postulated to induce the local release of cytokines, lipid mediators, and oxidants, resulting in pulmonary endothelial injury and increased-permeability lung edema $(8,9)$. While this hypothesis appears plausible, much of the supporting evidence is indirect and derives from comparison of pulmonary vascular responses between different species, which may possess significant differences other than the number of PIMs. This hypothesis would be strengthened if endotoxin sensitivity can be demonstrated to vary with PIM number within a given animal species. In a recent preliminary report (12), Longworth et al. have provided some support for this hypothesis by showing that 2-3-wkold lambs, which possess PIMs (13), had a larger pulmonary hemodynamic response to endotoxin compared with newborn lambs, which lack PIMs.

Within a given species, there is a significant variability in the individual's susceptibility to endotoxic injury. In species that are normally endotoxin resistant, one factor that has been

\footnotetext{
1. Abbreviations used in this paper: BDL, bile duct ligation; CSF-1, macrophage-colony stimulating factor; PIM, pulmonary intravascular macrophage.
} 
identified to correlate with enhanced endotoxin sensitivity is the presence of liver injury. Experimentally, D-galactosamine, which selectively suppresses hepatic RNA synthesis and causes acute hepatocellular injury, markedly sensitizes mice to the lethal effects of endotoxin (14) and enhances endotoxin-induced lung injury in rats (15). Moreover, Matuschak et al. have observed a high incidence of sepsis-induced acute lung injury in their patients with end-stage liver failure awaiting liver transplant (16). The mechanism of enhanced endotoxin sensitivity in liver disease is unknown, although it may relate in part to the depressed hepatic synthesis of acute phase proteins (17), or depressed hepatic metabolism of endogenous leukotrienes (15).

While studying the pulmonary circulatory changes in rats with liver cirrhosis due to chronic biliary obstruction $(18,19)$, we serendipitously observed large numbers of macrophage-like cells within the pulmonary microcirculation of these animals. While these cells morphologically resembled PIMs, it is unclear whether they are functionally active. In this study, we tested the hypothesis that chronic cholestatic liver injury induces the appearance of phagocytically active intravascular macrophages in rat lungs and studied the pathophysiological consequence of this PIM-induction. Cholestatic liver injury in rats was induced by surgical ligation of the common bile duct, which produced biliary cirrhosis in about $4 \mathrm{wk}$. Control rats received sham operation. At 1, 2, and $4 \mathrm{wk}$ after bile duct ligation (BDL), the presence of PIM-like cells were examined morphologically, by both light and electron microscopy, and functionally, by measuring lung uptake of fluorescent latex particles and ${ }^{99 \mathrm{~m}} \mathrm{Tc}-\mathrm{la}$ beled albumin colloids. To determine the potential pathophysiologic significance of this cellular alteration, the lethal and pulmonary edemagenic effects of endotoxin were compared in rats with biliary cirrhosis and sham-operated controls. In addition, pulmonary uptake of ${ }^{125} \mathrm{I}$-labeled endotoxin was quantitated in these rats. Our results indicate that chronic biliary obstruction induces the appearance of pulmonary intravascular phagocytes in rats and dramatically enhances their sensitivity to endotoxininduced lung vascular injury and death.

\section{Methods}

Animals and surgery. The following animal protocols have been reviewed and approved by the Animal Care and Use Committees of Northwestern University and Veterans Administration Lakeside Medical Center. Male, Sprague-Dawley rats (body wt 340-350 g) were purchased from Harlan Sprague Dawley, Inc. (Indianapolis, IN) and given free access to food and water. The animals were randomly assigned to either BDL or sham surgery (control). In all animals, laparotomy was performed under pentobarbital anesthesia $(50 \mathrm{mg} / \mathrm{kg}$, i.p.). In the BDL group, the common bile duct was isolated, double-ligated and up to a 6-mm section resected between the two ligatures as described by Kountouras et al. (20). The abdominal incision was then closed with sutures and surgical clips and the rats were allowed to recover. In the control group, the abdomen was closed following minimal manipulation of the abdominal content. BDL and control rats were then studied at 1,2 , or 4 wk after surgery.

Fluorescent microsphere uptake and electronmicroscopy. Fluorescein-labeled latex microspheres, $1 \mu \mathrm{m}$ in diameter, were purchased from Polysciences, Inc. (Warrington, PA) and used to document uptake by intravascular phagocytes in rat lungs (21). Nine BDL rats $(n=3$ each at 1, 2, and $4 \mathrm{wk}$ after BDL) and six control rats were studied. Each rat was injected with $0.1 \mathrm{ml}$ of fluorescent beads via tail vein and killed by a pentobarbital overdose $2 \mathrm{~h}$ later. The pulmonary artery was cannulated and the pulmonary vasculature was flushed free of circulating blood cells and excess beads with $30 \mathrm{ml}$ of a physiological salt solution containing $4 \mathrm{~g} / 100 \mathrm{ml}$ bovine serum albumin. The lungs are then quickly fixed by instillation of $2.5 \%$ glutaraldehyde into the airway at a constant pressure of $20 \mathrm{~cm} \mathrm{H}_{2} \mathrm{O}$. In two additional rats (one control and one BDL, $4 \mathrm{wk}$ ), the lungs were fixed by vascular perfusion (22) with cold glutaraldehyde solution at a mean pulmonary arterial pressure of $8 \mathrm{mmHg}$. After immersion in cold glutaraldehyde for $24 \mathrm{~h}$, small cubes of tissues were cut from each lobe, frozen in liquid nitrogen, and processed for cryostat sections. Cryostat sections were mounted directly onto glass slides and examined unstained for the presence of fluorescent microspheres using a fluorescent microscope. Random tissue blocks, 1$2 \mathrm{~mm}$ thick, were removed and post-fixed with $1 \% \mathrm{OsO}_{4}$, mounted on copper grids, and stained with $0.5 \%$ uranyl acetate. Transmission electronmicroscopy was performed using a Hitachi H600 electron microscope.

${ }^{99 m}$ Tc-Microlite uptake. Microaggregated albumin colloid (Microlite) with mean particle diameter of $1 \mu \mathrm{m}$ (95\% of particles are in range of 0.2-2 $\mu \mathrm{m}$ ) were purchased from E. I. du Pont (Billerica, MA), and labeled to ${ }^{99 \mathrm{~m}} \mathrm{Tc}$ according to the instructions provided by the manufacturer. Because of the short half-life of ${ }^{99 \mathrm{~m}} \mathrm{Tc}(6 \mathrm{~h})$, fresh ${ }^{99 \mathrm{~m}} \mathrm{Tc}$-Microlite was prepared on the morning of the experiment and used only for that day. Rats were injected with $\sim 1 \mu \mathrm{Ci}$ of ${ }^{99 \mathrm{~m}} \mathrm{Tc}$-Microlite via the tail vein. $60 \mathrm{~min}$ later, rats were killed by overdose with pentobarbital and samples of blood, lung, kidney, liver, and spleen were removed for determination of radioactivity. For each organ, the percentage uptake of ${ }^{99 \mathrm{~m}} \mathrm{Tc}-$ Microlite was calculated as follows: [(Total organ activity of $\left.{ }^{99 \mathrm{~m}} \mathrm{Tc}\right) /\left(\right.$ Total injected dose of $\left.\left.{ }^{99 \mathrm{~m}} \mathrm{Tc}\right)\right] \times 100$.

A total of 30 rats (17 BDL and 13 control) were studies in three separate experiments. 10 rats each were studied at 1 wk ( $6 \mathrm{BDL}, 4$ control), 2 wk ( 6 BDL, 4 control), and 4 wk ( 5 BDL, 5 control) after surgery. Because the results in control rats were similar regardless of the time after sham operation, the data from these 13 rats were pooled and analyzed as one group.

Effect of endotoxin on mortality and lung wet-to-dry weight ratio. $26 \mathrm{BDL}$ and 50 sham-operated control rats were studied at $4 \mathrm{wk}$ after surgery. Salmonella enteritidis LPS was injected intravenously $(0.01$, 0.1 , and $1 \mathrm{mg} / \mathrm{kg}$ in BDL rats; $0.01,0.1,1,10$, and $40 \mathrm{mg} / \mathrm{kg}$ in control rats) and the mortality was assessed over the next $72 \mathrm{~h}$. Rats that appeared moribund (i.e., unable to turn over when placed on their backs) were killed by pentobarbital overdose and considered as nonsurvivors. In these animals, the lung, liver, and spleen were removed and weighed at the time of death. Lung tissue was then dried in a Transite oven $\left(60^{\circ} \mathrm{C}\right)$ until constant weight, and the lung wet-to-dry weight ratio was calculated as an index of lung edema (23). Rats that remained alive at $72 \mathrm{~h}$ (survivors) were killed by pentobarbital overdose and their organs were removed and processed as above.

Lung uptake of ${ }^{125}$ I-endotoxin. Escherichia coli strain 0111:B4 LPS radiolabeled with iodine-125 (24) was kindly supplied by Dr. David Morrison (University of Kansas, Kansas City, KS). The specific activity of the preparation was $\sim 0.3 \mu \mathrm{Ci} / \mu \mathrm{g}$ LPS. ${ }^{125} \mathrm{I}-\mathrm{LPS}$ was diluted in saline containing $0.25 \%$ bovine serum albumin, and $\sim 1.7 \mu \mathrm{g}(\sim 5 \mu \mathrm{g} / \mathrm{kg})$ was injected into each rat via tail vein injection. After $4 \mathrm{~h}$, the rats were killed by pentobarbital overdose and their blood $(1 \mathrm{ml})$, liver, spleen, kidney, and lungs were removed for determination of radioactivity. The ${ }^{125}$ I-uptake in each organ was calculated as: [(Total organ ${ }^{125}$ I-activity )/ (Total injected dose of ${ }^{125}$ I-LPS)] $\times 100$.

Statistical analysis. Data are expressed as mean \pm SEM. Means of different experimental groups are analyzed using a one-way analysis of variance followed by multiple comparison with method of StudentNeuman-Keul to indicate pairs of groups with significant difference (25). When only two groups are involved, an unpaired $t$ test is used for statistical comparison. Linear correlations are calculated using standard methods (25). Differences in survival at each dose of endotoxin are compared using chi-square. Differences are considered significant when $P<0.05$.

\section{Results}

Within days after bile duct ligation, rats developed persistent jaundice associated with retardation of weight gain and progres- 
Table I. Organ Weights and ${ }^{99 m} T c-M i c r o l i t e ~ U p t a k e s$ after Bile Duct Ligation

\begin{tabular}{|c|c|c|c|c|}
\hline & \multirow[b]{2}{*}{ Control } & \multicolumn{3}{|c|}{ BDL } \\
\hline & & $1 \mathrm{wk}$ & 2 wk & 4 wk \\
\hline & $n=13$ & $n=6$ & $n=6$ & $n=5$ \\
\hline \multicolumn{5}{|l|}{ Body wt at } \\
\hline surgery (g) & $340 \pm 4$ & $351 \pm 3$ & $339 \pm 3$ & $351 \pm 2$ \\
\hline \multicolumn{5}{|l|}{ Body wt at } \\
\hline study (g) & $370 \pm 7$ & $322 \pm 11^{*}$ & $340 \pm 6 *$ & $338 \pm 14^{*}$ \\
\hline \multicolumn{5}{|l|}{ Organ wt (g) } \\
\hline Liver & $12.5 \pm 0.6$ & $16.8 \pm 1.0^{*}$ & $20.8 \pm 0.9 *$ & $21.1 \pm 2.2^{*}$ \\
\hline Spleen & $0.87 \pm 0.04$ & $0.92 \pm 0.08$ & $1.65 \pm 0.12 *$ & $2.38 \pm 0.31 *$ \\
\hline Kidney & $1.27 \pm 0.04$ & $1.16 \pm 0.03$ & $1.20 \pm 0.05$ & $1.54 \pm 0.18 *$ \\
\hline Lung & $1.47 \pm 0.03$ & $1.46 \pm 0.03$ & $1.63 \pm 0.06$ & $1.80 \pm 0.15^{*}$ \\
\hline Lung wet/dry & $5.14 \pm 0.11$ & $4.87 \pm 0.07$ & $4.84 \pm 0.10$ & $6.04 \pm 0.13^{*}$ \\
\hline \multicolumn{5}{|l|}{$\begin{array}{c}{ }^{99 \mathrm{~m}} \mathrm{Tc}-\text { Microlite } \\
\text { Uptake (\%) }\end{array}$} \\
\hline Liver $^{\ddagger}$ & $40.2 \pm 3.6$ & $45.2 \pm 3.3$ & - & $16.7 \pm 7.2^{*}$ \\
\hline Spleen $^{\ddagger}$ & $4.5 \pm 0.5$ & $3.1 \pm 0.4$ & - & $2.8 \pm 0.8^{*}$ \\
\hline Kidney & $1.3 \pm 0.1$ & $1.2 \pm 0.2$ & $1.3 \pm 0.1$ & $1.5 \pm 0.4$ \\
\hline Lung & $0.7 \pm 0.1$ & $1.7 \pm 0.2$ & $10.5 \pm 3.2^{*}$ & $35.1 \pm 5.9 *$ \\
\hline
\end{tabular}

Data are mean SEM; ${ }^{\ddagger}$ control data for liver and spleen ${ }^{99 \mathrm{~m}} \mathrm{Tc}$-Microlite uptake based on $n=9$. In the initial experiment involving the 2-wk group, only sections of the liver and spleen were saved for radioactivity measurement. As this was found to be inadequate for predicting total organ uptake, subsequent experiments in $1 \mathrm{wk}$ and $4 \mathrm{wk}$ rats were done with measurement of total liver and total spleen radioactivity. ${ }^{*} P$ $<0.05$ from control.

sive hepatosplenomegaly (Table I). The liver morphological and functional changes after BDL have been previously described $(20,26)$. At 4 wk after BDL, with liver cirrhosis present, the lung and kidney wet weights were significantly increased over those in sham-operated, control rats. Lung wet-todry weight ratio was also significantly increased at 4 wk after BDL (Table I).

Lung morphology and uptake of fluorescent beads. $2 \mathrm{~h}$ after intravenous injection, fluorescent beads were noted in lungs of BDL rats at 2 and 4 wk after bile duct ligation (Fig. $1 \mathrm{~B}$ ). These particles were sequestered in clusters within large cells in the alveolar septum (Fig. 1, C and D) No uptake was observed in the intra-alveolar cells. The lungs removed from BDL rats 1 wk after surgery showed only minimal, scattered lung uptake (not shown). No uptake of fluorescent beads was observed in the lungs of sham-operated, control rats (Fig. $1 \mathrm{~A}$ ).

On light microscopy, lungs from BDL rats showed accumulation of mononuclear cells within the lumen of small pulmonary vessels (Fig. 2). These cells were closely associated with the vascular endothelium and, in some, clusters of ingested latex beads were found within their abundant cytoplasm. Perivascular edema and alveolar septal thickening with increased cellularity were also observed in BDL rats, particularly at $4 \mathrm{wk}$ after the surgery (Fig. $2 B$ ).

Ultrastructural changes. On electron microscopy, lungs from sham-operated control rats contained normal cellular constituents with no macrophage-like cells seen in any of the sections examined (not shown). Lungs from BDL rats at 4-5 wk after surgery showed accumulation of large intravascular, mononuclear cells within capillaries and small vessels (Fig. 3). These cells had morphologic features of mature macrophages including indented nucleus, abundant cytoplasm containing phagocytic vacuoles, and many irregular surface projections (Fig. $3 \mathrm{~A}$ ). These cells were significantly larger than monocytes, some exceeding $30 \mu \mathrm{m}$ in maximal dimension. In addition, many of these cells exhibited large areas of close apposition with the underlying endothelial cells, including areas where the cytoplasm of the two cells interdigitate (Fig. $3 \mathrm{~B}$ ). However, junctional complexes of the kind described by Warner and Brain in sheep lungs (11), were not observed between these intravascular macrophage-like cells and endothelial cells. In many of these cells, ingested latex particles could be easily identified (Fig. 3, $A$ and $C$ ). Erythrophagocytosis by these phagocytes was also observed (Fig. $3 \mathrm{D}$ ).

In BDL rats at the earlier time point ( 1 and 2 wk), a large number of the intravascular cells were monocytes or cells with morphologic characteristics intermediate between monocytes and macrophages (not shown). With more advanced liver disease, some pulmonary microvessels appeared to be nearly obliterated by clusters of monocytes, neutrophils and intravascular macrophage-like cells (Fig. 3, $A$ and $C$ ). In these areas, ingested latex particles were found only within cytoplasms of the intravascular macrophage-like cells.

Quantitation of intravascular phagocytosis. Table I shows the ${ }^{99 \mathrm{~m}}$ Tc-Microlite uptake in various organs $1 \mathrm{~h}$ after intravenous injection. At 4 wk after BDL, despite increases in liver and spleen weight, the liver ${ }^{99 \mathrm{~m}} \mathrm{Tc}$-Microlite uptake was markedly decreased and the spleen uptake mildly decreased. There is a progressive increase in lung ${ }^{99 \mathrm{~m}} \mathrm{Tc}$-Microlite uptake after BDL, especially after the second week. ${ }^{99 m}$ Tc-Microlite uptake at 1 , 2 , and 4 wk after BDL were increased to 4-, 12-, and 47-fold of the corresponding values in time-matched, sham-operated control rats (Fig. 4). In contrast, ${ }^{99 \mathrm{~m}} \mathrm{Tc}$-Microlite uptake in the kidney was unchanged by BDL (Table I). In BDL rats, lung ${ }^{99 \mathrm{~m}} \mathrm{Tc}$-Microlite uptake correlated with increasing spleen weight, a reflection of the severity and chronicity of the portal hypertension associated with biliary cirrhosis (Fig. 5), and inversely with total ${ }^{99 \mathrm{~m}} \mathrm{Tc}-M i c r o l i t e ~ u p t a k e$ in the liver and spleen (not shown, $r=-0.79, P<0.001)$.

Endotoxin sensitivity. Table II shows the effect of $S$. enteritidis LPS on the $72 \mathrm{~h}$ mortality in control and BDL rats. As expected, control rats were highly resistant to endotoxin with $36 \%$ mortality at $10 \mathrm{mg} / \mathrm{kg}$ of LPS, i.v., and no death at $1 \mathrm{mg} /$ $\mathrm{kg}$ or lower doses of LPS. In contrast, LPS doses as low as 10 $\mu \mathrm{g} / \mathrm{kg}$ caused significant mortality in BDL rats. Overall, the LD50 for LPS was shifted to the left by at least two log orders of magnitude in chronic BDL rats.

Organ wet weights and lung wet-to-dry weight ratio were obtained in survivors at $72 \mathrm{~h}$ after endotoxin injection and in some nonsurvivors at the time of death (Table III). As expected, BDL rats showed marked hepatosplenomegaly compared to controls. Control rats dying after high doses of LPS (10-40 $\mathrm{mg} / \mathrm{kg}$ ) had normal wet lung weight and normal lung wet-todry ratio. However, BDL rats dying after low doses of LPS $(0.01-1 \mathrm{mg} / \mathrm{kg})$ had markedly increased wet lung weights and lung wet-to-dry ratio, indicative of fulminant pulmonary edema.

Lung uptake of ${ }^{125}$ I-LPS. Table IV shows the organ weights and ${ }^{125} \mathrm{I}$-LPS uptakes in control and cirrhotic rats $4 \mathrm{~h}$ after receiving an intravenous injection of ${ }^{125} \mathrm{I}$-LPS. As in previous experiments (Tables I and III), chronic BDL rats were charac- 

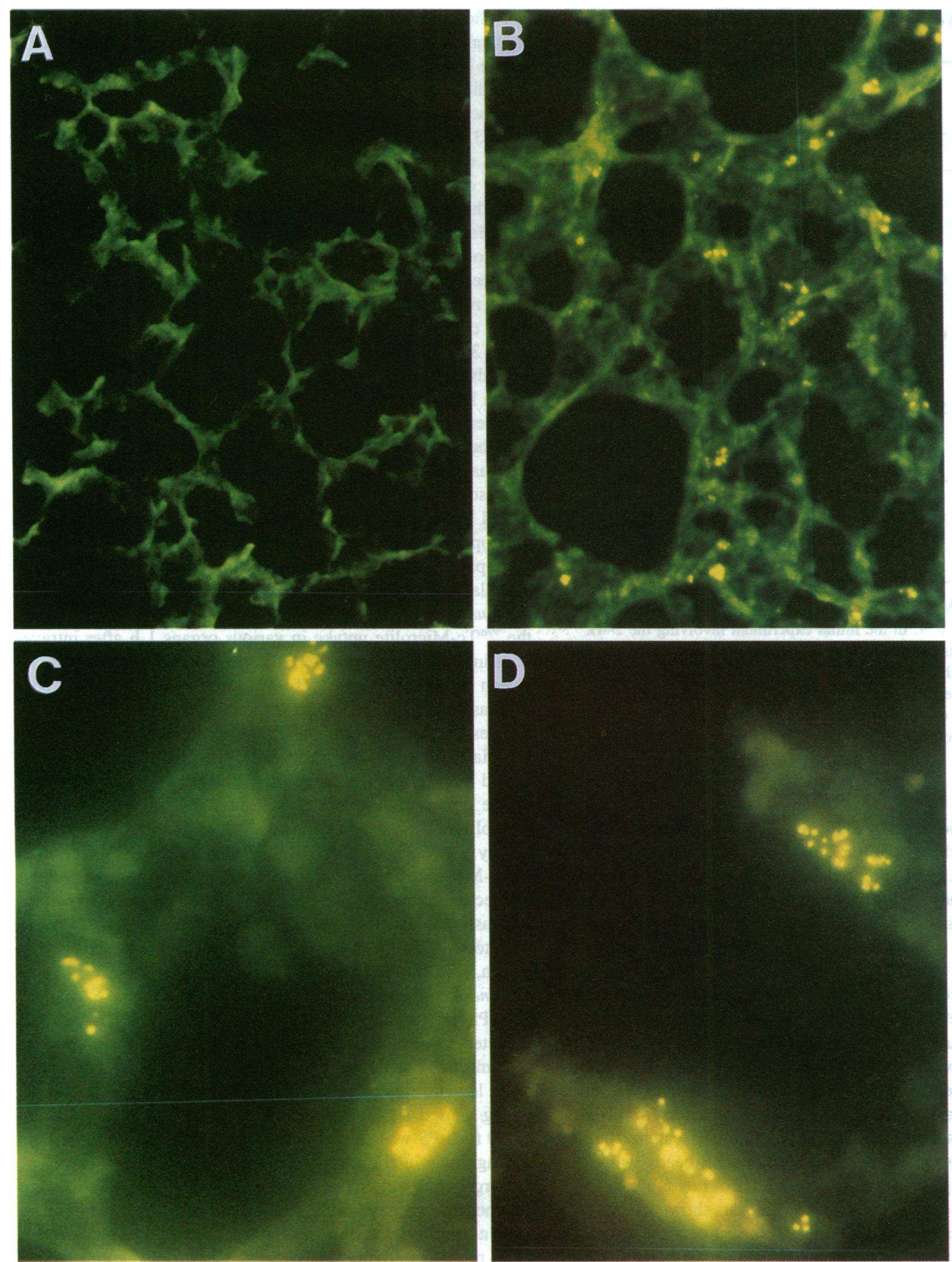

Figure 1. Pulmonary uptake of fluorescent microspheres after BDL $(A)$ Control rat lung (low power): Background fluorescence without any uptake of fluorescent latex particles. $(B)$ Lung section from BDL rats (4 wk, low power): Numerous clusters of fluorescent latex particles are retained within lung parenchyma. No uptake is observed in intraalveolar cells. $(C),(D)$ Lung sections from BDL rats (4 wk, high power): clusters of fluorescent particles within large interstitial/intravascular cells. 

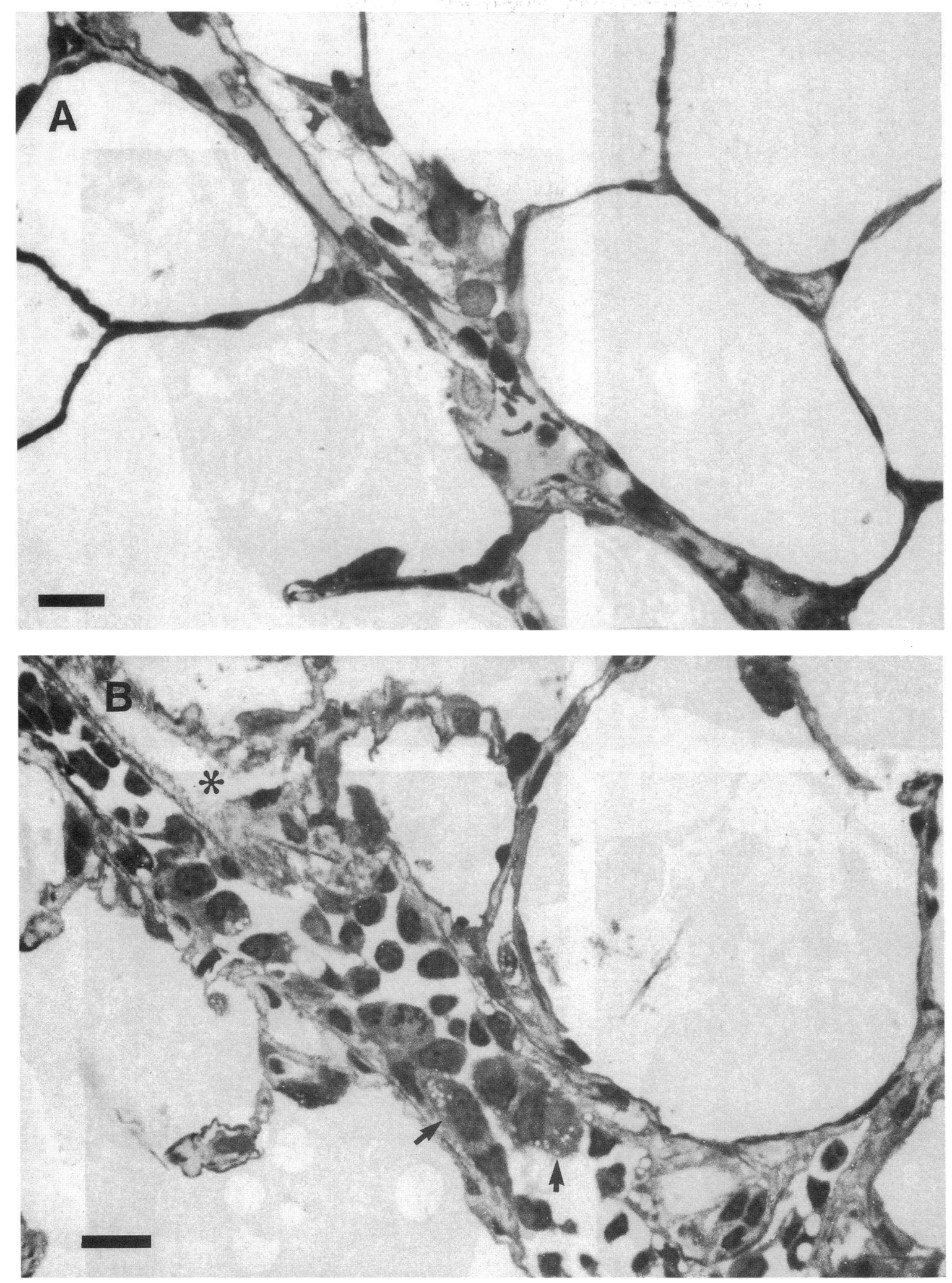

Figure 2. Light microscopic sections of lungs from BDL and control rats. Bar marker, $10 \mu \mathrm{m}$. $(A)$ Control rat lung: small vessel containing very few leukocytes. $(B)$ Lung section from BDL rat $(4 \mathrm{wk})$ : large number of leukocytes are observed in the vessel lumen. Two large, macrophagelike cells with ingested latex particles are present (arrow). There is also perivascular edema (star). 

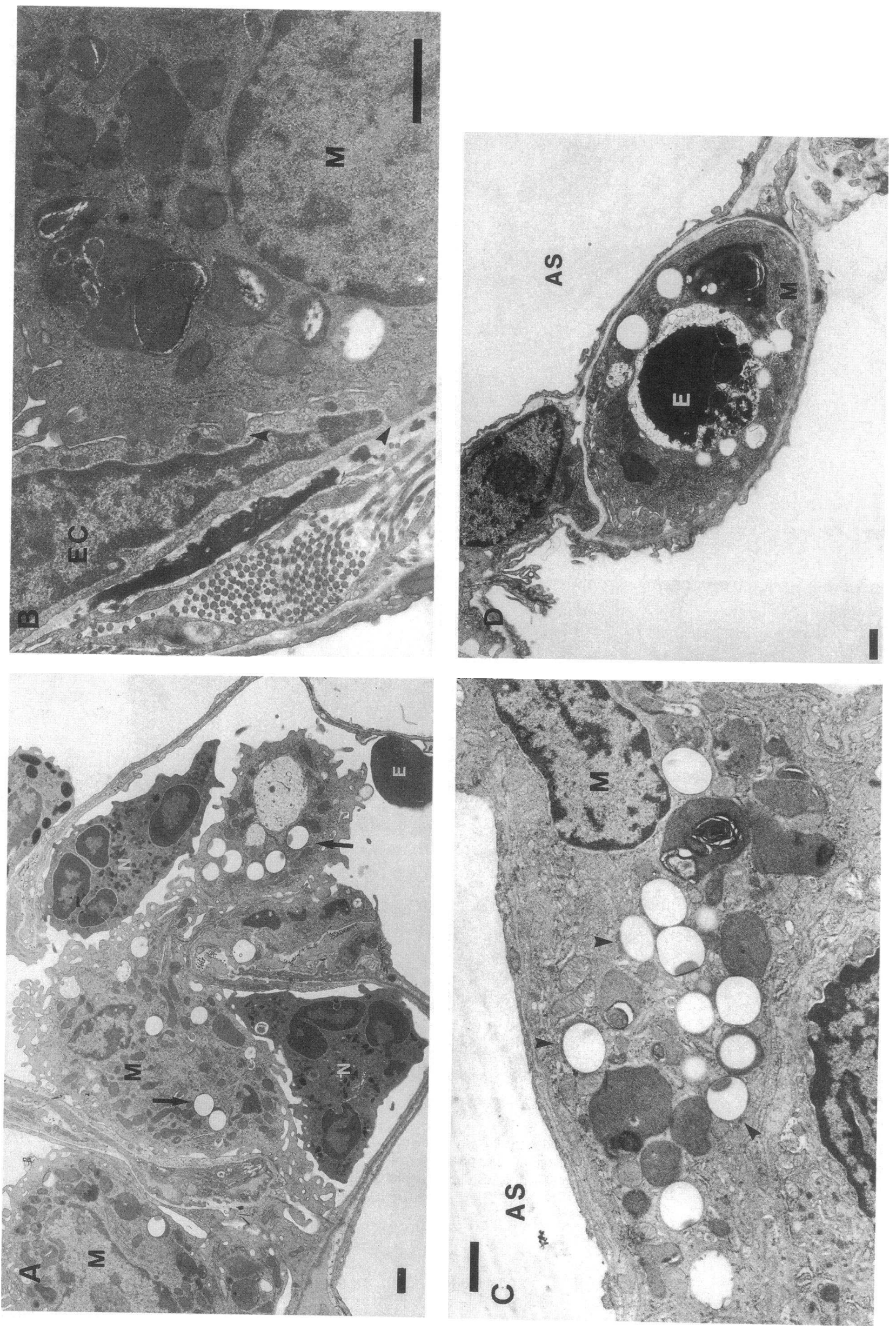


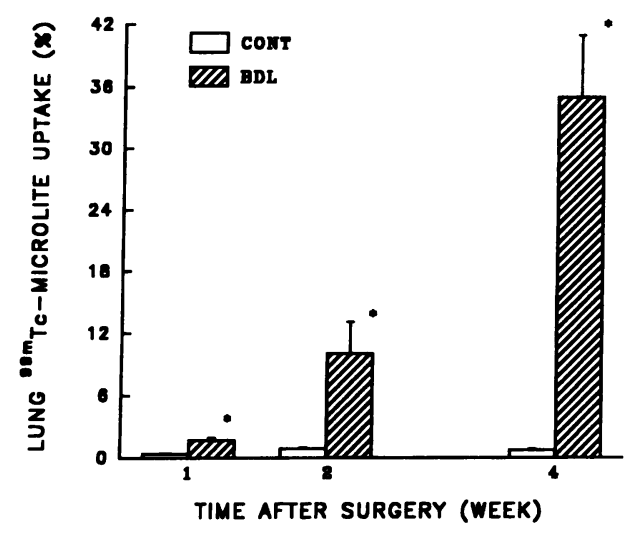

Figure 4. Lung ${ }^{99 \mathrm{~m}} \mathrm{Tc}$-Microlite uptake after BDL. Data shown are mean (and SE bar) lung uptake as a percentage of total injected dose of ${ }^{99 \mathrm{~m}} \mathrm{Tc}-$ microaggregated albumin (Microlite). Rats were studied at 1, 2, and 4 wk after either bile duct ligation (BDL) or sham-surgery (CONT). ${ }^{*} P$ $<0.05$ compared with control values at the corresponding time point.

terized by marked hepatosplenomegaly. While there were no changes in ${ }^{125}$ I-LPS uptakes in the liver and kidney, the lung ${ }^{125}$ I-LPS uptake was increased by over fivefold in BDL rats. In addition, following this very low dose of LPS $(\sim 5 \mu \mathrm{g} / \mathrm{kg})$, the wet lung weight in BDL rats was markedly increased when compared with the lung weight in either control rats given LPS (Table IV) or BDL rats not given LPS (Table I).

\section{Discussion}

While PIMs are normally not present in the lung circulation of some animal species, including the rat, several preliminary reports have suggested that, in these species, PIMs may be inducible (27-29). In this study, we demonstrated for the first time that intravascular macrophage-like cells appear in the pulmonary microcirculation of rats with chronic biliary obstruction, suggesting that cholestatic liver injury may be a reproducible stimulus for PIM induction in the rat. Our findings of markedly enhanced lung uptake of intravascular fluorescent latex beads, ${ }^{99}$ Tc-albumin colloids, and ${ }^{125}$ I-LPS in rats with biliary cirrhosis are consistent with the known function of PIM as an intravascular phagocyte. Moreover, the exquisite sensitivity of cirrhotic rats to endotoxin-induced lung edema and lethality suggests that the induction of these PIM-like cells is associated with potentially deleterious consequences for the host.

These large mononuclear cells found in the pulmonary microcirculation of rats with biliary cirrhosis exhibit many of the morphologic features previously described for PIMs in sheep and man. These features include the presence of an indented nucleus and abundant cytoplasm with irregular surface projections and containing lysomal granules, phagosomes, and phagolysosomes $(8,30)$. These macrophage-like cells can be distin-

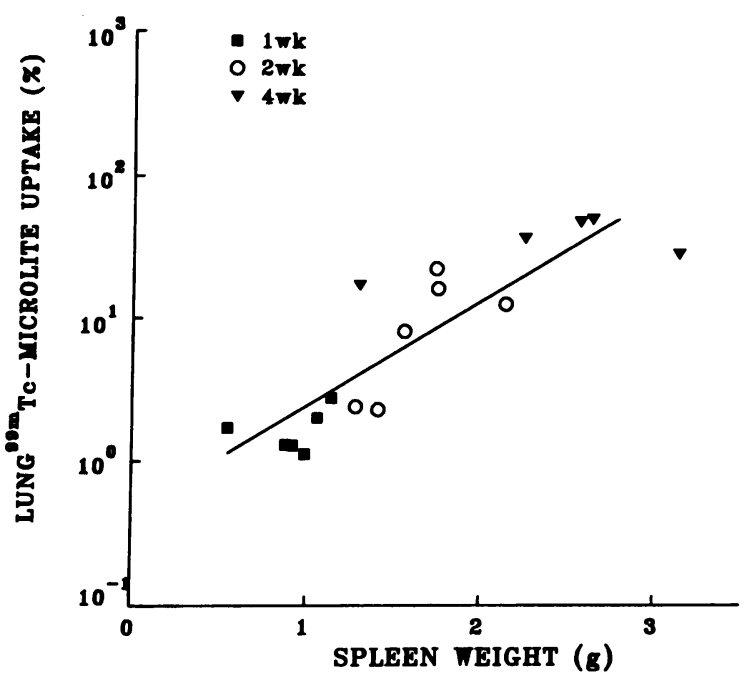

Figure 5. Correlation of lung ${ }^{99 \mathrm{~m}} \mathrm{Tc}$-Microlite uptake with spleen weight in BDL rats. Lung ${ }^{99 \mathrm{~m}} \mathrm{Tc}$-Microlite uptake, a measure of pulmonary intravascular phagocytosis, is significantly correlated with spleen weight, a reflection of the severity and chronicity of cholestatic liver disease. Regression line: Log (Lung ${ }^{99 \mathrm{~m}} \mathrm{Tc}$-Microlite Uptake) $=0.731$ (spleen wt) $-0.347 . r=0.87 ; P<0.01$.

guished from monocytes by their large size and by the presence of many ingested latex particles. Erythrophagocytosis, previously described in PIMs of calf and human lungs (30, 31), was also observed. In addition, these cells are closely attached to pulmonary endothelial cells, as demonstrated by areas of close apposition and interdigitation of their cell membranes. In contrast to what has been observed for PIMs in sheep or pigs $(8,31)$, but similar to a recent report in human lungs $(30)$, we did not find definitive evidence of adhesion complex with underlying endothelial cells.

Functionally, these cells resemble PIMs in their capacity for intravascular phagocytosis. Using a protocol described previously in the sheep (21), we compared the uptake of intravascular fluorescent latex particles by lungs of control and BDL rats. In agreement with previous reports (21), lungs from normal rats did not exhibit any uptake of these $1 \mu \mathrm{m}$-size particles. In contrast, lungs from BDL rats (at 2 and $4 \mathrm{wk}$ ) contained many cells with cluster of fluorescent latex beads within their cytoplasms. All of these bead-containing cells were located in the interalveolar septa, indicating that they are either intravascular or interstitial cells. Because of their large size, we would not expect these latex particles to traverse the alveolar capillary membrane and be engulfed by either interstitial or alveolar macrophages. Indeed, on light microscopy we did not observe particle uptake by alveolar macrophages in either control or cirrhotic rats. That other intravascular or interstitial phagocytes in the lung are not responsible for the increased particle uptake is

Figure 3. Electronmicrographs of lungs from BDL rats (4-5 wk). Bar marker, $1 \mu \mathrm{m}$. (A) Lung section following perfusion fixation: intravascular macrophages $(\mathrm{M})$, neutrophils $(\mathrm{N})$ and erythrocyte $(\mathrm{E})$ within a dilated microvessel. Only the intravascular macrophages contain ingested latex particles (arrow). (B) High power electronmicrograph showing areas of interdigitation (arrow) between the cytoplasm of an intravascular macrophage (M) and an endothelial cell (EC). (C) Ingestion of latex particles (arrow) by an intravascular macrophage (M) which fills the entire capillary space. AS, air space; E, erythrocyte. $(D)$ Erythrophagocytosis by an intravascular macrophage (M) in a pulmonary capillary. AS, air space; E, erythrocyte. 
Table II. Endotoxic Mortality in Bile Duct-Ligated and Control Rats

\begin{tabular}{llllll}
\hline & \multicolumn{5}{c}{ LPS (mg/kg) } \\
\cline { 2 - 5 } Group & 0.01 & 0.1 & 1 & 10 & 40 \\
\hline Control & $0 / 10(0 \%)$ & $0 / 10(0 \%)$ & $0 / 11(0 \%)$ & $5 / 14(36 \%)$ & $5 / 5(100 \%)$ \\
BDL & $2 / 6(33 \%)$ & $7 / 11(64 \%)^{*}$ & $4 / 9(44 \%)^{*}$ & - & - \\
\hline
\end{tabular}

S. enteritidis LPS was injected intravenously and mortality at $72 \mathrm{~h}$ is shown as No. died/No. tested (percent mortality). $\quad * P<0.05$ compared to control value by chi-square.

supported by our electron microscopic study in which clusters of latex beads are found only in the intravascular mononuclear cells exhibiting many of the morphologic characteristics of PIMs.

To quantitate pulmonary intravascular phagocytosis, the rats were injected intravenously with ${ }^{99 \mathrm{~m}} \mathrm{Tc}$-labeled Microlite and their lungs were removed for measurement of radioactivity $1 \mathrm{~h}$ later. Microlite is a colloidal preparation of microaggregated albumin which has a mean diameter of about $1 \mu \mathrm{m}$. After intravascular injection, it is readily taken up by cells of the reticuloendothelial system. Because of its ease of preparation, ${ }^{99} \mathrm{~m}$ TcMicrolite has been advocated as a superior agent to ${ }^{99 \mathrm{~m}} \mathrm{Tc}$-sulfur colloid for liver-spleen scan in humans (32). As expected, Microlite was rapidly taken up by the liver and spleen in control rats with less than $1 \%$ of the injected radioactivity recovered in lung tissue. However, following bile duct ligation, there was a progressive increase in lung ${ }^{99 \mathrm{~m}} \mathrm{Tc}-\mathrm{Microlite}$ uptake, to 12 and 47 folds of the values in sham-operated controls at 2 and 4 wk after BDL, respectively. Furthermore, lung ${ }^{99 \mathrm{~m}}$ Tc-Microlite uptake correlated with the severity of liver disease as reflected in the increased spleen weight and the decreased total liver and spleen phagocytic activities. While lung ${ }^{99 \mathrm{~m}} \mathrm{Tc}$-Microlite uptake may not directly reflect the actual number of PIMs, our results certainly indicate marked enhancement of lung intravascular phagocytic activity in rats with cholestatic liver disease. In fact, the level of lung particle uptake in some of our cirrhotic rats approaches the level observed previously in sheep lungs (21).

In addition to the increased phagocytosis of intravascular latex beads and albumin colloids, lungs from rats with biliary cirrhosis also demonstrate increased uptake of ${ }^{125}$ I-LPS. While
LPS is a much smaller molecule, it binds to plasma high density lipoprotein and is cleared from the blood stream by cells of the reticuloendothelial system (33). Previous studies have consistently demonstrated increased lung uptake of labeled LPS in animals with PIMs $(8,9)$. In normal rats, LPS was not detectable in lung tissue using histochemical technique during the first $7 \mathrm{~h}$ after intravenous injection (33), and we found only $0.37 \%$ of the injected ${ }^{125}$ I-LPS in lungs of control rats after 4 h. In rats with biliary cirrhosis, the lung ${ }^{125}$ I-LPS uptake was increased fivefold, to a level which is approximately $38 \%$ of the total liver uptake. In addition to being consistent with the presence of a significant population of PIMs in these rats, this impressive increase in lung LPS uptake also suggests the possibility of enhanced pulmonary toxicity during endotoxemia.

In view of the many studies demonstrating marked endotoxin sensitivity in animals with large numbers of PIMs (810 ), we wondered if chronic BDL rats, which acquired these PIM-like cells, would become endotoxin sensitive. Indeed this was what we found. While control rats tolerated high doses (up to $1 \mathrm{mg} / \mathrm{kg}$ i.v.) of endotoxin without any obvious adverse effects, chronic BDL rats appeared ill with lethargy and piloerection with as little as $0.01 \mathrm{mg} / \mathrm{kg}$ of endotoxin. Moreover, rats with biliary cirrhosis exhibited high mortality rates with very low doses $(0.01-1 \mathrm{mg} / \mathrm{kg})$ of endotoxin. These results are consistent with the recent findings of Kleber et al. that rats with secondary biliary cirrhosis are more sensitive to the hemodynamic effects of $E$. coli endotoxin (34).

In addition to the enhanced sensitivity to the lethal effect of endotoxin, rats with biliary cirrhosis became highly susceptible to endotoxin-induced lung edema. In species with large

Table III. Comparisons of Body and Organ Weights and Lung Wet-to-Dry Weight Ratio in Survivors and Nonsurvivors after LPS

\begin{tabular}{|c|c|c|c|c|c|c|}
\hline Group & & Body wt & Liver & Spleen & Lung & Lung W/D \\
\hline & $n$ & $g$ & $g$ & $g$ & $g$ & \\
\hline \multicolumn{7}{|l|}{ Control } \\
\hline Survivor & (17) & $348 \pm 6$ & $11.9 \pm 0.2$ & $1.2 \pm 0.1$ & $1.80 \pm 0.06$ & $4.52 \pm 0.09$ \\
\hline Nonsurvivor & (6) & $362 \pm 10$ & $14.1 \pm 1.0$ & $1.1 \pm 0.1$ & $1.61 \pm 0.09$ & $4.69 \pm 0.16$ \\
\hline \multicolumn{7}{|l|}{ BDL } \\
\hline Survivor & (10) & $365 \pm 4$ & $20.4 \pm 0.8^{\ddagger}$ & $2.42 \pm 0.2^{* \neq}$ & $1.82 \pm 0.05$ & $(4.59)$ \\
\hline Nonsurvivor & (8) & $364 \pm 14$ & $29.6 \pm 2.0^{* \pm}$ & $3.1 \pm 0.3^{* \neq}$ & $3.87 \pm 0.33^{* \ddagger}$ & $6.53 \pm 0.40^{* \ddagger}$ \\
\hline
\end{tabular}

Data are mean \pm SEM. Control and BDL rats were given various doses of LPS intravenously (see Table II) and separated into those that were alive at $72 \mathrm{~h}$ (Survivors) and those that died before $72 \mathrm{~h}$ (Nonsurvivors). When rats appeared moribund, or at $72 \mathrm{~h}$, the rats were given an overdose of pentobarbital and their liver, spleen, and lung were removed and weighed. Nonsurvivors in control group died at $5.8 \pm 0.2 \mathrm{~h}$ (mean $\pm \mathrm{SE}$ ) after $10-40 \mathrm{mg} / \mathrm{kg}$ of LPS while nonsurvivors in BDL group died at $2.7 \pm 0.2 \mathrm{~h}$ after $0.01-1 \mathrm{mg} / \mathrm{kg}$ of LPS. In some rats (control/survivor, $n=11$; control/nonsurvivors, $n=5$; BDL/survivor, $n=1$; BDL/nonsurvivor, $n=6$ ), lung wet-to-dry weight ratios were obtained. $* P<0.05$ from control/ survivor; ${ }^{\ddagger} P<0.05$ from control/nonsurvivors. 
Table IV. Organ Weights and ${ }^{125}$ I-LPS Uptakes in Control and BDL Rats

\begin{tabular}{lcc}
\hline & Control $(n=5)$ & BDL, 4 wk $(n=5)$ \\
\hline Birth wt at study (g) & $403 \pm 8$ & $344 \pm 8^{*}$ \\
Organ Weight $(\mathrm{g})$ & & \\
$\quad$ Liver & $13.2 \pm 0.5$ & $23.0 \pm 2.3^{*}$ \\
Spleen & $1.05 \pm 0.06$ & $2.79 \pm 0.38^{*}$ \\
Kidney & $2.45 \pm 0.06$ & $2.84 \pm 0.11^{*}$ \\
Lung & $1.84 \pm 0.03$ & $3.05 \pm 0.30^{*}$ \\
${ }^{125}$ I-LPS uptake (\%) & & \\
Blood & $0.38 \pm 0.06$ & $0.45 \pm 0.08$ \\
Liver & $5.4 \pm 0.9$ & $5.4 \pm 0.7$ \\
Spleen & $0.33 \pm 0.06$ & $0.91 \pm 0.14^{*}$ \\
Kidney & $0.43 \pm 0.07$ & $0.66 \pm 0.08$ \\
Lung & $0.37 \pm 0.05$ & $2.06 \pm 0.61^{*}$ \\
& & \\
\hline
\end{tabular}

Data are mean \pm SEM. Organ wet weights and ${ }^{125}$ I-LPS uptakes (expressed as $\%$ of the total injected dose) were measured at $4 \mathrm{~h}$ after intravenous injection of ${ }^{125}$ I-LPS. Blood "uptake" indicates the amount of residual radioactivity (percentage of injected dose) per $\mathrm{ml}$ of whole blood. $* P<0.05$ from control rats.

numbers of PIMs, endotoxin causes marked pulmonary hypertension, and increased-permeability lung edema (2). In contrast, although high doses of endotoxin ( $1-20 \mathrm{mg} / \mathrm{kg}$ ) consistently increase pulmonary vascular permeability in the rat $(4,35)$, it has been difficult to demonstrate significant lung edema in these animals even after lethal doses of endotoxin $(4,7)$. In this study, we found that rats with biliary cirrhosis given $0.01-1$ $\mathrm{mg} / \mathrm{kg}$ of LPS had extremely heavy lungs at the time of death while control rats dying after $10-40 \mathrm{mg} / \mathrm{kg}$ of endotoxin had normal or low wet lung weights. The measurement of lung wetto-dry weight ratio in cirrhotic rats confirmed the presence of lung edema following endotoxin exposure. Interestingly, as little as $5 \mu \mathrm{g} / \mathrm{kg}$ of endotoxin (during the ${ }^{125}$ I-LPS study) acutely increased the wet lung weights in rats with biliary cirrhosis. Based on previous studies in other species discussed above (811 ), it is likely that these PIM-like cells are responsible for the enhanced sensitivity to endotoxin-induced lung injury in the cirrhotic rats. It has been postulated that the release of protein cytokines, arachidonic acid-derived eicosanoids, and plateletactivating factor by endotoxin-stimulated macrophages in the pulmonary microcirculation (36) initiates the cascade of pathophysiologic events culminating in acute lung injury.

An important question is whether an analogous process of PIM-induction occurs in patients with liver cirrhosis. It is generally accepted that PIMs are not present in normal human lungs (37). However, PIM-like cells have been observed in human lung specimen resected for a variety of noninfectious indications (30), suggesting that PIMs may be inducible in man. While there is currently no data regarding the presence or absence of intravascular macrophages in lung tissue from patients with liver disease, several reports in the nuclear medicine literature have suggested that hepatic dysfunction may be associated with pulmonary intravascular phagocytosis in humans (38-40). Keyes et al. reported a group of 22 patients who demonstrated increased lung uptake of ${ }^{99 \mathrm{~m}} \mathrm{Tc}$-sulfur colloid during liver spleen scan (38). The increased lung uptake occurred in the absence of increased bone marrow uptake, and was felt not to be due to technical factors such as microembolization. Interestingly, all of these patients had some form of liver disease and their prognosis was extremely poor. Furthermore, in experimental studies, induction of acute or chronic liver injury by carbontetrachloride have also been noted to increase lung uptake of colloids in rats (41-42). Taken together, these data suggest that in humans and rats, whose reticuloendothelial systems are normally concentrated in the liver and spleen, hepatic dysfunction enhances pulmonary intravascular phagocytosis. These results suggest that this shift in reticuloendothelial activity is due to an induction of a mononuclear cell population in the lung microcirculation that resembles in both morphology and function the PIM described in sheep and pigs.

Although not specifically addressed in this study, one may speculate that the presence of large numbers of PIM-like cells could contribute to other pulmonary circulatory alterations in liver cirrhosis. In humans, cirrhosis is associated with a loss or attenuation of hypoxic pulmonary vasoconstriction (43) and increased intrapulmonary shunting (44), resulting in variable degrees of arterial hypoxemia. We have recently noted similar changes in rats with chronic bile duct ligation-induced cirrhosis (18). Furthermore, lungs from chronic BDL rats also exhibited increased vascular permeability and elevated thromboxane B2 level (19), findings that are indicative of microvascular injury. It is possible that these intravascular phagocytes, perhaps stimulated by circulating endotoxins derived from the gut (45), contribute to the early demise of bile duct-ligated rats by causing thromboxane-dependent pulmonary endothelial cell injury and lung edema (19). Furthermore, phagocytosis of circulating bacteria and/or endotoxin by PIMs $(8,9)$ may account for the high incidence of sepsis-induced adult respiratory distress syndrome in patients with end-stage liver disease (16).

The cellular origin and mechanism of induction of these intravascular macrophage-like cells in rats with biliary cirrhosis are unclear. Based on detailed studies in other species (46), as well as our recent findings of increased numbers of lung and peripheral blood monocytes in rats with early stages of biliary obstruction (47), it is reasonable to assume that these PIMs are derived from circulating monocytes. The presence of cells with morphology intermediate between monocytes and macrophages in lung sections from rats with early stages of biliary obstruction further supports this hypothesis. An alternative possibility based on anatomic studies by Schneeberger-Keeley and Burger in cats (48), and Patek and Bernick in rabbits (49) is the migration of liver Kupffer cells to the pulmonary microcirculation which in their study occurred following intravenous administration of colloidal carbon (49). However, the same authors have noted that there was much less Kupffer cell migration in rats given the same phagocytic challenge (50). In a preliminary study using three BDL rats, in which the liver Kupffer cells were labeled with fluorescent latex beads prior to bile duct ligation, very few labeled Kupffer cells were found within the pulmonary microcirculation at $4 \mathrm{wk}$. The majority of lung intravascular phagocytes, demonstrated by uptake of iron oxide particles injected $1 \mathrm{~h}$ before killing, contained no fluorescent beads. (S. W. Chang and N. Ohara, unpublished observation). Thus, it appears that Kupffer cell migration plays a relatively minor role in the appearance of pulmonary intravascular phagocytes in rats with liver cirrhosis. Other alternatives such as increased proliferation of pulmonary resident macrophages are even less likely given the low proliferating potential of the differentiated 
macrophages (51), and the lack of resident intravascular macrophage population in the normal rat.

One potential mechanism which may account for the development of PIM-like cells in chronic liver disease is endogenous endotoxemia. Circulating endotoxin has been detected in the blood of patients with liver cirrhosis (45) and in rats after bile duct ligation (52). Moreover, administration of endotoxin has been reported to increase pulmonary intravascular phagocytosis $(53,54)$ and to induce monocyte retention in rabbit lungs $(55)$ and the appearance of PIM-like cells in rats (27). While we have recently confirmed the observation that endotoxemia acutely increases pulmonary intravascular phagocytosis, we feel that this is due to sequestered neutrophils and activated monocytes in the pulmonary microcirculation (S. W. Chang, unpublished observation). In fact, we have been unable to document any increase in lung uptake of either fluorescent latex beads or ${ }^{99 \mathrm{~m}} \mathrm{Tc}$-Microlite in rats following chronic injections of $S$. enteritidis LPS for up to 4 wk (S. W. Chang, unpublished observations). Thus, it is our feeling that chronic endotoxemia alone can not account for the induction of PIM-like cells in this setting.

Other potential mechanisms for the induction of PIM-like cells in liver cirrhosis are even more speculative, but include alterations in the synthesis or metabolism of various cytokine growth factors which may promote monocyte migration, adherence, or differentiation. One such factor is the macrophagecolony simulating factor (CSF-1), a potent growth factor which induces macrophage proliferation and differentiation (56). A recent preliminary report has documented elevated CSF-1 content in human liver biopsy specimen (57). In addition, monocytes isolated from peripheral blood of cirrhotic patients exhibited in vitro evidence of activation with excessive production of tumor necrosis factor and interleukin 6 in response to LPS (58) and spontaneous release of platelet-derived growth factor (59). Thus, chronic inflammatory liver injury could lead to an overproduction of one or more of these cytokines, which then stimulate the proliferation and maturation of mononuclear phagocytes into tissue macrophages. Why this process should result in a preferential accumulation of intravascular macrophages in the lung is unclear.

In summary, we have demonstrated that chronic biliary obstruction in rats results in the appearance of large numbers of intravascular macrophage-like cells in the pulmonary microcirculation. The induction of these PIM-like cells is associated with pulmonary intravascular phagocytosis and increased sensitivity to endotoxic lung injury and death. These data provide strong support for the hypothesis that PIMs account for the intraspecies, as well as the interspecies, variability in endotoxin sensitivity. Furthermore, it is proposed that the induction of PIMs in patients with liver disease may account for their enhanced susceptibility to sepsis-induced lung injury (16). The validity of this hypothesis will need to be tested in future clinical studies.

\section{Acknowledgments}

We thank Dr. D. Morrison, University of Kansas, for providing the radiolabeled endotoxin, Jan Henson (National Jewish Center for Immunology and Respiratory Medicine) and Jim Anderson (Dr. Lewis Smith's laboratory at Veterans Administration Lakeside Medical Center) for assistance with electron microscopy, and Mario Cruz for typing the manuscript. Xin Wang and Heather Rossi provided valuable technical assistance.
Supported by grants from the National, Heart, Lung, and Blood Institute (Clinical Investigator Award HL-01966 to S. Chang), the Department of Veterans Affairs (Associate Investigator Award to N. Ohara and Merit Review Award to S. Chang) and the Chicago Lung Association. S. Chang is a Career Investigator of the American Lung Association.

\section{References}

1. Berczi, I., L. Bertok, and T. Bereznai. 1966. Comparative studies on the toxicity of Escherichia coli lipopolysaccharide endotoxin in various animal species. Can. J. Microbiol. 12:1070-1071.

2. Brigham, K. L., and B. Meyrick. 1986. Endotoxin and lung injury. Am. Rev. Respir. Dis. 133:913-927.

3. Olson, N. C., T. T. Brown, Jr., and D. L. Anderson. 1985. Dexamethasone and indomethecin modify endotoxin-induced respiratory failure in pigs. $J$. Appl. Physiol. 58:294-284.

4. Chang, S., C. O. Fedderson, P. M. Henson, and N. F. Voelkel. 1987. Platelet-activating factor mediates hemodynamic changes and lung injury in endotoxin-treated rats. J. Clin. Invest. 79:1498-1509.

5. Guenter, C. A., V. Fiorica, and L. B. Hinshaw. 1969. Cardiorespiratory and metabolic responses to live $E$. coli and endotoxin in the monkey. J. Appl. Physiol. 26:780-786.

6. Welsh, C. H., I. M. Dauber, and J. V. Weil. 1986. Endotoxin increases pulmonary vascular protein permeability in the dog. J. Appl. Physiol. 61:13951402.

7. Rinaldo, J. E., J. H. Dauber, J. Christman, and R. M. Rogers. 1984. Neutrophil alveolitis following endotoxemia. Enhancement by previous exposure to hyperoxia. Am. Rev. Respir. Dis. 130:1065-1071.

8. Warner, A. E., and J. D. Brain. 1990. The cell biology and pathogenetic role of pulmonary intravascular macrophages. Am. J. Physiol. 258:L1-L12.

9. Warner, A. E., M. M. DeCamp, R. M. Molina, and J. D. Brain. 1988. Pulmonary removal of circulating endotoxin results in acute lung injury in sheep. Lab. Invest. 59:219-230.

10. Dehring, D. J., and B. J. Wismar. 1989. Animal models of sepsis. In The Pulmonary Intravascular Macrophage. N. C. Staub, editor. Futura Publishing Co., Inc., Mount Kisco, NY. 79-100.

11. Warner, A. E., and J. D. Brain. 1986. Intravascular pulmonary macrophages: a novel cell removes particles from blood. Am. J. Physiol. 250:R728R732.

12. Longworth, K. E., A. Westgate, and N. C. Staub. 1991. Development of pulmonary hemodynamic and lymph dynamic responses to endotoxin in newborn lambs. FASEB (Fed. Am. Soc. Exp. Biol.) J. 5, A1429. (Abstr.).

13. Longworth, K. E., A. M. Westgate, M. K. Grady, J. Y. Westcott, and N. C. Staulb. 1992. Development of pulmonary intravascular macrophage function in newborn lambs. J. Appl. Physiol. 73:2608-2615.

14. Galanos, C., M. A. Freudenberg, and W. Reutter. 1979. Galactosamineinduced sensitization to the lethal effect of endotoxin. Proc. Natl. Acad. Sci. USA. 76:5939-5943.

15. Matuschak, G. M., M. R. Pinsky, E. C. Klein, D. H. Van Thiel, and J. E. Rinaldo. 1990. Effects of D-galactosamine-induced acute liver injury on mortality and pulmonary responses to $E$. coli lipopolysaccharide. Am. Rev. Respir. Dis. 141:1296-1306.

16. Matuschak, G. M., J. E. Rinaldo, M. R. Pinsky, J. D. Gavaler, and Van Thiel. 1987. Effect of end-stage liver failure on the incidence and resolution of the adult respiratory distress syndrome. J. Crit. Care. 2:162-173.

17. Alcorn, J. M., J. Fierer, and M. Chojkier. 1992. The acute-phase response protects mice from D-galactosamine sensitization to endotoxin and tumor necrosis factor-a. Hepatology. 15:122-129.

18. Chang, S., and N. Ohara. 1992. Pulmonary circulatory dysfunction in rats with biliary cirrhosis: An animal model of the hepatopulmonary syndrome. Am. Rev. Respir. Dis. 145:798-805.

19. Chang, S., and N. Ohara. 1993. Increased pulmonary vascular permeability in rats with biliary cirrhosis: Role of thromboxane A2. Am. J. Physiol. 264:L245L252.

20. Kountouras, J., B. H. Billing, and P. J. Scheuer. 1984. Prolonged bile duct obstruction: a new experimental model for cirrhosis in the rat. Br. J. Exp. Pathol. 65:305-311

21. Wheeldon, E. B., and J. H. Hansen-Flaschen. 1986. Intravascular macrophages in the sheep lung. J. Leukocyte Biol. 40:657-661.

22. Bachofen, H., A. Ammann, D. Wangensteen, and E. R. Weibel. 1982. Perfusion fixation of lungs for structure-function analysis: credits and limitations. J. Appl. Physiol. 53:528-533.

23. Pearce, M. L., J. Yamashita, and J. Beazell. 1965. Measurement of pulmonary edema. Circ. Res. 16:482-488.

24. Ulevitch, R. T. 1978. The preparation and characterization of a radiolabeled bacterial lipopolysaccharide. Immunochemistry. 15:157-164. 
25. Steel, R. G. D., and J. H. Torrie. 1960. Principles and Procedures of Statistics. McGraw-Hill Book Co., Inc., New York.

26. Ohara, N., and J. Reichen. 1986. Aminopyrine breath test predicts changes in hepatocyte microsomal function but not hepatocyte mass in bile duct ligated cirrhotic rats. Hepatology. 6:1206 (Abstr.).

27. Warner, A. E., M. M. DeCamp, C. F. Bellows, and J. D. Brain. 1989. Endotoxemia enhances lung uptake of circulating particles in species lacking pulmonary intravascular macrophages. Am. Rev. Respir. Dis. 139:A158 (Abstr.).

28. Cooper, J. A., B. K. McCandless, T. J. Ferro, and A. B. Malik. 1988 Pulmonary intravascular phagocytic activity in rabbits and sheep. Am. Rev. Respir. Dis. 137:398A (Abstr.).

29. Fracica, P. J., T. Bertram, M. Knapp, and J. D. Crapo. 1988. Pulmonary intravascular macrophages in normal and oxygen injured baboon lung tissue. Clin. Res. 36:591A (Abstr.).

30. Dehring, D. J., and B. L. Wismar. 1989. Intravascular macrophages in pulmonary capillaries of humans. Am. Rev. Respir. Dis. 139:1027-1029.

31. Winkler, G. C. 1988. Pulmonary intravascular macrophages in domestic animal species: Review of structural and functional properties. Am. J. Anat. 181:217-234.

32. Saha, G. B., D. H. I. Feiglin, J. K. O’Donnell, R. T. Go, P. M. Karam, and W. McIntyre. 1986. Experience with Technetium ${ }^{-99} \mathrm{~m}$ albumin colloid kit for reticuloendothelial imaging. J. Nucl. Med. Technol. 14:149-151.

33. Freudenberg, M. A., N. Freudenberg, and C. Galanos. 1982. Time course of cellular distribution of endotoxin in liver, lungs and kidneys of rats. Br. J. Exp. Pathol. 63:56-65.

34. Kleber, G., A. Braillon, C. Gaudin, B. Champigneulle, S. Cailmail, and D. Lebrec. 1992. Hemodynamic effects of endotoxin and platelet-activating factor in cirrhotic rats. Gastroenterology. 103:282-288.

35. Chang, S., J. Y. Wescott, W. C. Pickett, R. C. Murphy, and N. F. Voelkel. 1989. Endotoxin-induced lung injury in rats: role of eicosanoids. J. Appl. Physiol. 66:2407-2418

36. Bertram, T. A., L. H. Overby, R. Danilowicz, T. E. Eling, and A. R. Brody. 1988. Pulmonary intravascular macrophages metabolize arachidonic acid in vitro. Am. Rev. Respir. Dis. 138:936-944.

37. Gehr, P., M. Bachofen, and E. R. Weibel. 1978. The normal human lung. Ultrastructure and morphometric estimation of diffusion capacity. Respir. Physiol. 32:121-140.

38. Keyes, J. W. Jr., G. A. Wilson, and J. D. Quinones. 1973. An evaluation of lung uptake of colloid during liver imaging. J. Nucl. Med. 14:687-691.

39. Klingensmith, W. C., III, S. L. Yang, and H. N. Wagner, Jr. $1978 .{ }^{99 \mathrm{~m}} \mathrm{Tc}$ sulfur colloid in liver and spleen imaging. J. Nucl. Med. 19:31-35.

40. Imarisio, J. J. 1975. Liver scan showing intense lung uptake in neoplasia and infection. J. Nucl. Med. 16:188-190.

41. Paumgartner, G., J. Longueville, and C. M. Leevy. 1968. Phagocytic activity in experimental liver injury. Exp. Mol. Pathol. 9:161-170.

42. Noda, T., H. Mimura, and K. Orita. 1990. Assessment of Kupffer cell function in rats with chronic liver injury caused by CCI4. Hepato-gastroenterology. 37:319-323.

43. Daoud, F. S., J. T. Reeves, and J. W. Schaefer. 1972. Failure of hypoxic pulmonary vasoconstriction in patients with liver cirrhosis. J. Clin. Invest. 51:1076-1080.

44. Krowka, M. J., and D. A. Cortese. 1990. Hepatopulmonary syndrome: an evolving perspective in the era of liver transplantation. Hepatology. 11:138-142.

45. Prytz, H., J. Holst-Christensen, B. Korner, and H. Liehr. 1976. Portal venous and systemic endotoximia in patients with cirrhosis. Scand. J. Gastroenterol. 11:857-863.

46. Winkler, G. C., and N. F. Cheville. 1985. Monocytic origin and postnatal mitosis of intravascular macrophages in the porcine lung. J. Leukocyte Biol. 38:471-480.

47. Chida, M., PHS Sporn, X. Wand, and S. Chang. 1993. Perfusion isolation of pulmonary intravascular macrophages from rats with biliary cirrhosis. Am. Rev. Respir. Dis. (Abstr.). 147:A307.

48. Schneeberger-Keeley, E. E., and E. J. Burger, Jr. 1970. Intravascular macrophages in cat lungs after open chest ventilation. Lab. Invest. 22:361-369.

49. Patek, P. R., and S. Bernick. 1960. Time sequence studies of reticuloendothelial cell responses of foreign particles. Anat. Rec. 138:27-37.

50. Frankel, H. H., P. R. Patek, and S. Bernick. 1962. Long term studies of the rat reticuloendothelial system and endocrine gland responses to foreign particles. Anat. Rec. 142:359-373.

51. Van Furth, R. 1982. Current view on the mononuclear phagocyte system. Immunobiology. 161:178-185.

52. Gouma, D. J., J. C. U. Coelho, J. D. Fisher, J. F. Schlegel, Y. F. Li, and F. G. Moody. 1986. Endotoxemia after relief of biliary obstruction by interna and external drainage in rats. Am. J. Surg. 151:476-479.

53. Quinones, J. D. 1973. Localization by technetum-sulfur colloid after RES stimulation. J. Nucl. Med. 14:443-444.

54. Klingensmith, W. C., III, and V. J. Lovett, Jr. 1974. Lung uptake of ${ }^{99} \mathrm{mTc}$ sulfur colloid secondary to intraperitoneal endotoxin. J. Nucl. Med. 15:10281031.

55. Ohgami, M., C. M. Doerschuk, R. P. Gie, D. English, and J. C. Hogg. 1992. Late effect of endotoxin on the accumulation and function of monocytes in rabbit lungs. Am. Rev. Respir. Dis. 146:190-195.

56. Sherr, C. J. 1990. Regulation of mononuclear phagocyte proliferation by colony-stimulating factor-1. Int. J. Cell Cloning. 8(Suppl. 1):46-60.

57. Esposti, S. D., R. Stanley, and M. A. Zern. 1990. Macrophage-colony stimulating factor (CSF-1) content is markedly increased in human liver disease. Hepatology. 12:908 (Abstr.).

58. Deviere, J., J. Content, C. Denys, P. Vandenbussche, L. Schandene, J. Wybran, and E. DuPont. 1990. Excessive in vitro bacterial lipopolysaccharideinduced production of monokines in cirrhosis. Hepatology. 11:628-634.

59. Peterson, T. C., and R. A. Isbrucker. 1992. Fibroproliferation in liver disease: Role of monocyte factors. Hepatology. 15:191-197. 